\title{
La institución psiquiátrica en Colombia en el año 2025. Investigación con método Delphi*
}

\section{Mental health institution in Colombia in 2025. Delphi Method}

\section{A instituição psiquiátrica na Colômbia no ano 2025. Pesquisa com o método Delphi}

Fecha de recepción: 22-06-15 Fecha de aceptación: 24-08-15 Disponible en línea: 01-11-15 doi: 10.11144/Javeriana.rgyps14-29.ipci

Cómo citar este artículo:

Vanegas Medina CR, de la Espriella Guerrero RA. La institución psiquiátrica en Colombia en el año 2025. Investigación con método Delphi. Rev. Gerenc. Polít. Salud. 2015; 14(29): 114-135. http://dx.doi. org/10.11144/Javeriana.rgyps14-29.ipci

Claudia Rocío Vanegas-Medina**

Ricardo Andrés de la Espriella-Guerrero***

Declaración de apertura: investigación no patrocinada por industria farmacéutica, subvencionada por los autores.

** Médico cirujan de la Universidad Javeriana, especialista en Psiquiatría de la Universidad Nacional de Colombia, especialista en Docencia Universitaria de la Universidad El Bosque, médico psiquiatra del Hospital Santa Clara y de la Clínica de Nuestra Señora de la Paz. Docente de posgrado de Psiquiatría en la Fundación Universitaria Juan N Corpas, profesor asistente en la Universidad El Bosque. Correo electrónico: claudiarociovanegas@hotmail.com. Correspondencia: Clínica Nuestra Señora de La Paz, Avenida Centenario 68F 25, Bogotá.

*** Médico y cirujano de la Universidad Javeriana, médico especialista en Psiquiatría de la Universidad Javeriana, terapeuta sistémico, Sistemas Humanos, Kensington Consultation Centre. Magíster en Epidemiología Clínica de la Pontificia Universidad Javeriana, docente del Departamento de Psiquiatría, Facultad de Medicina de la Pontificia Universidad Javeriana. Gestor de docencia e investigación en la Clínica Nuestra Señora de La Paz, Bogotá. Correo electrónico: rde@javeriana.edu.co. 


\section{Resumen}

La incertidumbre de las instituciones psiquiátricas en Colombia tiene aspectos científicos, económicos, de mercado y regulatorios que dificultan la previsión de las decisiones para una atención adecuada, adaptada a las condiciones cambiantes de la prestación de salud. La investigación exploró cómo será la institución psiquiátrica en el año 2025, con metodología Delphi. Hallazgos: Existe gran incertidumbre de cómo adecuar la institución psiquiátrica en el futuro; de acuerdo con los cambios de legislación y las expectativas sociopolíticas en el momento del estudio. Se prevé la necesidad de educación del equipo profesional, mejoría en infraestructura e incremento de servicios, incluyendo consulta, hospitalización total y parcial, clínicas específicas y atención comunitaria. La institución ideal luce como un conjunto amplio de servicios desarrollados sobre la base de la prestación actual, asumiendo pacientes agudos y crónicos. El campo de acción de la clínica psiquiátrica se prevé continuará asumiendo la falta de desarrollo de la atención primaria y de dispositivos comunitarios.

Palabras clave: hospitales psiquiátricos; salud mental; psiquiatría; atención psiquiátrica; investigación sobre servicios de salud; método Delphi

\section{Abstract}

Uncertainty of psychiatric institutions in Colombia has scientific, economic, market and regulatory aspects that hinder the provision of decisions for adequate, efficient and effective care, facing the changing conditions of the provision of health services. The research explores how psychiatric institution will be in 2025. Materials and Methods: Delphi methodology, quantitative and qualitative analysis. Findings: There is great uncertainty about how to adapt the psychiatric institution in the future; according with changes in legislation and sociopolitical expectations at time of study. Education of professional team, improved infrastructure and increased services, including ambulatory ones, total and partial hospitalization, specific clinical and community care is expected. The ideal institution looks like a comprehensive set of services developed on the basis of the current provision, assuming acute and chronic patients. The scope of the psychiatric clinic is expected to continue assuming the lack of development of primary care and community facilities.

Keywords: psychiatric hospitals; mental health; psychiatry; psychiatric care; health services research; Delphi method

\section{Resumo}

A incerteza das instituições psiquiátricas na Colômbia tem aspectos científicos, económicos, de mercado e regulatórios que dificultam a previsão das decisões para um atendimento adequado, adaptado às condições variáveis da prestação de saúde. A pesquisa explorou como poderia ser a instituição psiquiátrica no ano 2025, com metodologia Delphi. Achados: Existe grande incerteza sobre como adequar a instituição psiquiátrica no futuro, de acordo com as mudanças da legislação e as expectativas sociopolíticas no momento do estudo. Prevê-se a necessidade de educação da equipe profissional, melhoramento da infraestrutura e acréscimo de serviços, incluindo consulta, hospitalização total e parcial, clínicas específicas e atendimento comunitário. A instituição ideal aparece como uma vasta gama de serviços desenvolvidos sobre a base da prestação atual, assumindo pacientes agudos e crônicos. O campo de ação da clínica psiquiátrica prevê-se continuando a assumir a falta de desenvolvimento no atendimento primário e de dispositivos comunitários.

Palavras-chave: hospitais psiquiátricos; saúde mental; psiquiatria; atendimento psiquiátrico; pesquisa sobre serviços de saúde; método Delphi 


\section{Justificación}

La prestación adecuada de atención en salud mental supone una previsión por parte de los diferentes actores involucrados de las adecuaciones del modelo de prestación de servicio en el futuro, incluyendo el desarrollo de instituciones, planes de beneficios, normatividad prevista y costos de atención (1). Esta situación es evidente en las instituciones de salud mental; en nuestro país se han cerrado clínicas (en el 2013 la clínica San Juan de Dios de Chía, con 260 camas) y disminuido servicios de atención en salud mental en hospitales generales (en Bogotá programas de hospital Día para Niños, unidades de salud mental en hospital general), lo que evidencia una necesidad mayor de un modelo que anticipe, en lo posible, los cambios del entorno.

Aspectos tales como la recesión económica mundial, los costos de la atención en salud mental, el envejecimiento poblacional, el aumento de enfermedades crónicas, la disponibilidad de tratamientos nuevos y costosos, junto con una legislación más centrada en resultados y una demanda mayor de los usuarios de acceso ilimitado a la atención en salud, son temas que preocupan a las instituciones; se busca una planeación de servicios que den respuesta a las necesidades de los pacientes, familias, comunidades, profesionales y gobierno $(2,3)$.

La Organización Mundial de la Salud (oms) ha enfatizado en la necesidad de una respuesta integral y coordinada de los sectores sanitario y social, evidenciando la complejidad de la atención que no puede ser cubierta solamente por el sector salud $(2,4)$. La oms en el plan de acción 2013-2020 fija cuatro objetivos principales: liderazgo y gobernanza más eficaces en materia de salud mental; prestación de servicios integrales e integrados de salud mental y asistencia social con base en la comunidad; aplicación de estrategias de promoción prevención; y fortalecimiento de los sistemas de información, evidencia e investigación (2).

En Colombia, en 1974 se creó la División de Salud Mental del Ministerio de Salud (5); posteriormente, la Política Nacional del Campo de la Salud Mental (2007) intentó articular la atención psiquiátrica con la perspectiva de derechos y la política pública (610), estableciendo los objetivos, principios, líneas de acción y estrategias específicas (11), aunque no tuvieron el desarrollo e impacto esperado. En 2013 se promulgó la Ley de Salud Mental (Ley 1616 de 2013) (12), de la cual se esperaba que tuviera el impacto que anteriores medidas no tuvieron, mediante una adecuada implementación (13).

Diversas razones posicionan la salud mental como fundamental para la salud pública, como la prevalencia de trastornos mentales, estimada en dos de cada cinco adultos, que afecta a todas las clases sociales y países, asî como su alto costo tanto económico como emocional (14). Las acciones de salud mental deben incluir a los individuos, familias y comunidades (15), no limitadas al campo específico de salud, tales como son los determinantes sociales de la enfermedad.

\section{Epidemiología}

Colombia es uno de los países con mayor prevalencia de trastornos mentales y una menor proporción de uso de los servicios (16). Las proyecciones estadísticas muestran que las condiciones psiquiátricas y neurológicas a nivel mundial se incrementarán de 10,5\% del total de la carga de la enfermedad a $15 \%$ en el año 2020 (17). Se prevé que en el año 2015 en Colombia la depresión unipolar será la primera causa general de consulta (15). 
En Colombia en el año 2003, 40,1\% de la población entre 18 y 65 años presentaba un trastorno psiquiátrico diagnosticado (14), siendo los trastornos de ansiedad los más frecuentes $(19,5 \%)$, seguidos de los trastornos del estado de ánimo $(13,3 \%)$, los trastornos por control de impulsos $(9,3 \%)$ y los relacionados con el consumo de sustancias psicoactivas $(9,4 \%)$; estos últimos afectan en forma importante a población joven (14).

Al comparar la prevalencia de los trastornos mentales en Colombia con otros países, se encuentra que el país ocupa el segundo puesto en el trastorno por control de impulsos, el cuarto puesto en los trastornos de ansiedad y en los relacionados con el consumo de sustancias psicoactivas y el quinto puesto en los trastornos del estado de ánimo (17).

\section{Institución psiquiátrica: historia}

El primer hospital psiquiátrico europeo fue construido en 1409 en Valencia (18). El hospital mental ha sido cuestionado permanentemente, buscando un manejo más humano y científico (18). La antipsiquiatría y otros movimientos han cuestionado las prácticas psiquiátricas, el diagnóstico y la institución mental (19), permeando el discurso oficial de la psiquiatría $(5,10)$, así como el discurso de las ciencias sociales.

Se ha propuesto acabar con el hospital mental (19), integrar la atención psiquiátrica al hospital general (15) y centrarse en promoción y prevención; sin embargo, la institución mental se ha reconvertido, para adaptarse a las prácticas, políticas y avances terapéuticos (15).

Se recomienda hacer el menor uso posible de hospitales psiquiátricos, desplazando la atención hacia estructuras comunitarias más flexibles (15). En Colombia hay dificultad para llevar a cabo el modelo de desinstitucionalización, ya que la prestación de servicios de salud en general está centrada en las actuaciones profesionales.

Las organizaciones de salud, incluyendo las psiquiátricas, se consideran empresas, con regulaciones propias de mercado y procesos administrativos complejos (20), por lo cual se impone una necesidad de innovación y transformación de los esquemas de atención médica tradicionales, de cara a condiciones tales como la mayor utilización de los servicios médicos, las restricciones en la oferta de camas hospitalarias y de especialistas, los costos de tratamiento y el envejecimiento de la población (21).

\section{Marco legal}

En Colombia la Constitución de 1991 consagró la salud como servicio público y derecho social $(14,22)$; posteriormente, la Ley 100 de 1993 (23) fijó las condiciones de la prestación de la atención en salud, en tanto que la Ley 1616 (12) estableció las condiciones específicas en salud mental, en los ítems de: prevención, atención integral, red integral de servicios en salud mental, atención ambulatoria, atención domiciliaria, atención prehospitalaria, centros de atención en drogadicción y servicios de farmacodependencia, centros de salud mental comunitaria, grupos de apoyo de pacientes y familias, hospital de día para adultos, hospital de día para niñas, niños y adolescentes, rehabilitación basada en comunidad, unidades de salud mental, urgencias de psiquiatría y atención integral a niños, niñas y adolescentes.

\section{Método Delphi}

El método Delphi es un método cualitativo, se inició en el Centro de Investigación de RAND 
Corporation, como un instrumento para realizar predicciones sobre un caso de catástrofe nuclear (24). Esta metodología ha sido utilizada como un sistema para obtener información sobre el futuro en diversas áreas tales como salud, economía, administración, etc.

La técnica Delphi se define como un método de estructuración de un proceso de comunicación grupal efectivo, al permitir a un grupo de individuos, como un todo, tratar un problema complejo (24). El método Delphi incluye la selección de un grupo de expertos a los que se les pregunta su opinión sobre cuestiones referidas a acontecimientos del futuro, en sucesivas rondas, anónimas, con máxima autonomía por parte de los participantes. La capacidad de predicción de este método se basa en la utilización sistemática de un juicio intuitivo emitido por un grupo de expertos (25).

\section{Materiales y métodos}

Metodología Delphi tradicional (25), con consulta a expertos y rondas sucesivas de preguntas.

Fase 1: formulación del problema. Se define el problema que se va a estudiar, en general, como "La salud mental en Colombia para el año 2025", dividiéndolo en tres grandes aspectos: la institución psiquiátrica, el ejercicio profesional del psiquiatra y la formación en psiquiatría en el año 2025. Se presenta la primera fase de la investigación, centrada en la institución psiquiátrica en el año 2025.

Elaboración de encuesta: se realiza una encuesta, de acuerdo con los aspectos contemplados en la Ley de Salud Mental (Ley 1616 de 2013 o Ley Esperanza) (12), validando el contenido y la comprensión de la encuesta con grupos de profesionales que no se incluyeron entre los expertos consulta- dos. Dadas las respuestas de la primera fase por parte de los expertos, se agruparon los dominios de análisis, incluyendo la atención primaria y la psiquiatría comunitaria en una sola categoría.

Los dominios considerados fueron:

- Hospitalización

- Consulta externa

- Hospitalización parcial

- Unidad de salud mental

- Especialización de servicios

- Atención domiciliaria

Otros dominios de la investigación correspondientes a la formación en psiquiatría y a la especialidad no se presentan en este artículo. Se elaboró el cuestionario y se envió enlace por internet en www.encuesta.e.

Fase 2: elección de expertos. Se buscó representatividad de los expertos por tipo de institución (pública, privada, experiencia docente y área de trabajo específico), que fueran médicos psiquiatras, docentes de universidades tanto públicas como privadas, con experiencia clínica y algunos con experiencia en investigación. El muestreo se limitó a la ciudad de Bogotá.

Se realizó una primera aproximación a los expertos elegidos $(n=22)$, en la cual se indagó sobre el interés de participar. Una vez establecido el contacto, se solicitó a cada experto autorización para su ingreso al estudio y permiso para utilizar su nombre en la lista de expertos consultados.

Fase 3: desarrollo metodológico. Se realizaron dos fases de preguntas, una inicial y 
la segunda de acuerdo con los resultados que requirieron concertación o explicación, profundizando en conceptos.

Fase 4: análisis de resultados, cuantitativo y cualitativo. Se realizó la transcripción a Excel de las respuestas, con tabulación de resultados cuantitativos y análisis de discurso de comentarios y ampliación de las respuestas, categorizando estas últimas.
Se elaboró un plantilla para análisis cualitativo de la forma descrita en la figura 1.

\section{Resultados}

Se contactó a 22 expertos según las condiciones del estudio. A la encuesta inicial ingresaron 19 expertos, aunque algunos de ellos no contestaron ninguna pregunta. Debido a dificultades de continuidad y limitaciones de la encuesta en línea, se realizó

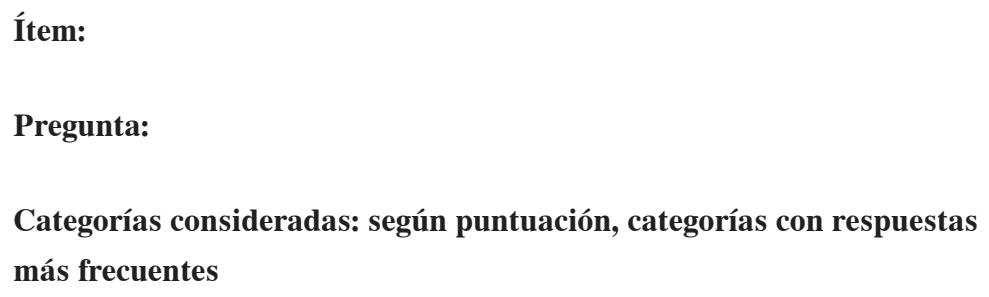

- Selección de datos contenidos en la categoría:

- Descripción:

- Relación entre variables:

- Revisión de los datos:

- Posibles explicaciones:

- Conceptualización más general (patrón cultural):

- Formulación tentativa de nuevas hipótesis:

- Búsqueda de nuevos hallazgos en los datos:

\section{Comentarios:}


contacto directo con cada experto a través de correo electrónico.

Número de personas segunda ronda: 14, de los cuales ampliaron las repuestas solicitadas 12 de ellos; por tanto, el análisis de las preguntas que requirieron consenso se realiza sobre esos 12. Se deja esta muestra, dado que el método permite entre doce y treinta expertos (24).

La tendencia prevista de los programas de salud mental para el año 2025 se presenta en la tabla 1.

Los expertos puntuaron todos los problemas listados como susceptibles de atención primaria en salud mental, atención en unidades de salud mental de hospital general, hospitalización parcial y atención hospitalaria en clínica psiquiátrica (adicciones comportamentales, adicciones farmacológicas, ansiedad, trastorno afectivo bipolar, déficit de atención, demencia, depresión, esquizofrenia, intentos de suicidio, retardo mental, trastornos adaptativos, trastornos de aprendizaje, trastornos de conducta, trastornos de la conducta alimentaria, trastornos de la personalidad, trastornos por estrés, trastornos profundos del desarrollo); ninguna otra opción fue añadida a la lista.

La atención psiquiátrica en servicios especializados en salud mental (clínica psiquiátrica) deberá incluir farmacodependencia (programa de desintoxicación) y larga estancia (80\%), seguida por trastornos de la alimentación $(73,3 \%)$, trastornos de la conducta $(66,67 \%)$, inimputabilidad $(60 \%)$, retardo mental $(60 \%)$ y epilepsia y trastornos relacionados $(53,3 \%)$.

La institución ideal en psiquiatría para el año 2025 deberá contener todos los servicios enumerados (atención domiciliaria, atención psicoterapéutica de pareja y familia, atención psicoterapéutica individual, centro de empleo, clínica de memoria, consulta externa psiquiátrica especializada, consulta externa psiquiátrica general, hospitalización 24 horas en unidad de agudos, hospitalización domiciliaria, hospitalización parcial (día, noche), programas de adicciones, rehabilitación cognoscitiva, servicios de integración comunitaria, telepsiquiatría, unidad de niños y adolescentes). No se nombraron opciones adicionales.

La atención en salud mental en clínicas especializadas para trastornos específicos deberá desarrollarse en todas las condiciones establecidas en el cuestionario (abuso sexual, adicciones, clínicas de trastornos bipolares, clínicas de trastornos de ansiedad, clínicas de trastornos de la conducta alimentaria, clínicas de trastornos de la personalidad y discontrol de impulsos, clínicas de trastornos depresivos, clínicas de trastornos esquizofrénicos, clínicas de trastornos por déficit cognoscitivo, clínicas de trastornos relacionados con estrés, déficit cognitivo, demencias y trastornos relacionados, dolor, epilepsia, maltrato y violencia intrafamiliar, psicogeriatría, suicidio, trastornos de aprendizaje, trastornos de la conducta, trastornos del desarrollo, trastornos relacionados con el estrés); ninguno de los expertos consultados anotó otras opciones.

Ante la afirmación "Algunos autores opinan que la atención de larga estancia para pacientes con trastornos mentales severos refractarios a cargo del estado para el año 2025 pasará a ser manejada por instituciones particulares", los expertos respondieron afirmativamente en $83,3 \%$ la opción "Será manejada tanto por instituciones privadas como públicas"; $16,7 \%$ la opción “será manejada exclusivamente por instituciones privadas", 8,3\% la opción "No será asumida ni por instituciones públicas o privadas" y ninguno marcó la opción "Será asumida totalmente por instituciones públicas". Tanto 


\begin{tabular}{|c|c|c|c|c|c|c|}
\hline 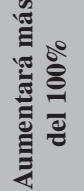 & 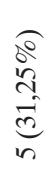 & $\begin{array}{l}\widehat{d} \\
m \\
m \\
\stackrel{m}{\vec{v}}\end{array}$ & $\begin{array}{l}\text { of } \\
\stackrel{m}{2} \\
\stackrel{2}{2}\end{array}$ & 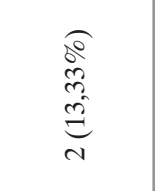 & $\underset{m}{\stackrel{d}{d}}$ & $\begin{array}{l}\widehat{d} \\
0 \\
0 \\
0 \\
0 \\
0\end{array}$ \\
\hline 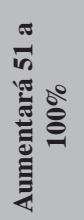 & $\begin{array}{l}\frac{\partial}{2} \\
\frac{n}{n} \\
\infty \\
\stackrel{\infty}{=}\end{array}$ & $\begin{array}{l}\text { od } \\
\stackrel{m}{m} \\
\stackrel{n}{2}\end{array}$ & 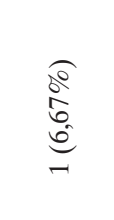 & $\begin{array}{l}\hat{\sigma} \\
m \\
\stackrel{m}{2} \\
\stackrel{n}{2}\end{array}$ & $\begin{array}{l}\widehat{\partial} \\
\text { m } \\
\stackrel{\sim}{\vec{d}}\end{array}$ & $\begin{array}{l}\text { वे } \\
\text { ले } \\
\text { d্ } \\
\vec{\sigma}\end{array}$ \\
\hline 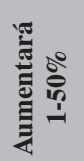 & 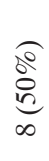 & $\begin{array}{l}\widehat{d} \\
m \\
m \\
\hat{n} \\
\infty\end{array}$ & \begin{tabular}{l}
20 \\
$\frac{8}{6}$ \\
\multirow{6}{0}{} \\
0
\end{tabular} & 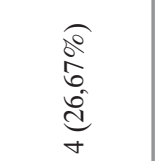 & $\frac{\widehat{d}}{0}$ & $\begin{array}{l}0 \\
0 \\
0 \\
0\end{array}$ \\
\hline 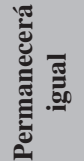 & $\begin{array}{l}\text { of } \\
\text { ê } \\
0\end{array}$ & $\frac{\text { o }}{\stackrel{d}{m}}$ & 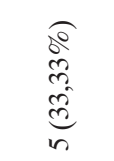 & 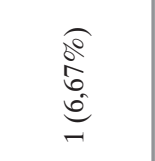 & $\frac{\text { od }}{\stackrel{d}{d}}$ & $\begin{array}{l}\widehat{\rho} \\
0 \\
0 \\
\hat{\infty} \\
\stackrel{-}{\infty}\end{array}$ \\
\hline 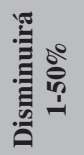 & $\begin{array}{l}\text { of } \\
\text { e} \\
0\end{array}$ & $\frac{\widehat{d}}{\stackrel{0}{e}}$ & $\frac{8}{8}$ & $\begin{array}{l}\widehat{d} \\
m \\
m \\
m \\
m \\
m\end{array}$ & $\begin{array}{l}\widehat{80} \\
\hat{6} \\
0 \\
-1\end{array}$ & $\frac{\widehat{d}}{e_{0}^{2}}$ \\
\hline 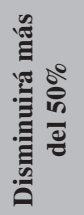 & $\frac{\widehat{d e}}{e_{0}^{2}}$ & $\stackrel{d}{e}_{0}^{d}$ & $\frac{d}{8}$ & $\begin{array}{l}\widehat{8} \\
60 \\
0 \\
0\end{array}$ & $\frac{\widehat{d}}{e_{0}^{2}}$ & $\frac{\widehat{d}}{\stackrel{0}{e}}$ \\
\hline 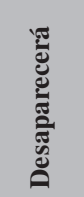 & $\frac{\widehat{d}}{e_{0}^{2}}$ & $\stackrel{d}{e}_{0}^{\frac{d}{e}}$ & $\frac{8}{8}$ & $\frac{\widehat{d}}{e_{0}^{2}}$ & $\frac{\widehat{d}}{e_{0}^{2}}$ & $\stackrel{d}{e}_{0}^{0}$ \\
\hline 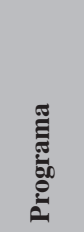 & 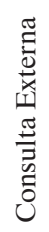 & 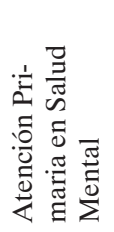 & 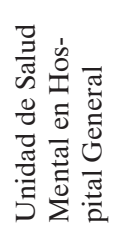 & 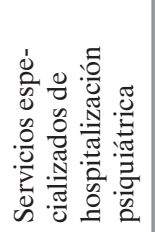 & 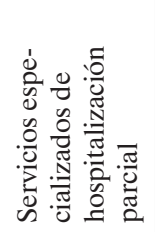 & 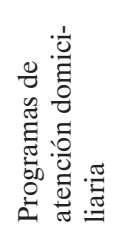 \\
\hline
\end{tabular}

Rev. Gerenc. Polit. Salud, Bogotá (Colombia), 14 (29): 114-135, julio-diciembre de 2015 
la factibilidad como la probabilidad de que la respuesta anterior se diera puntuaron entre 3 y 5 , siendo 3 y 5 cada uno con $25 \%$ y 4 con $50 \%$.

En cuanto a los programas de atención en el domicilio se prevé que se aplicarán en mayor medida para depresión, trastornos por estrés y esquizofrenia, con $91,7 \%$ de respuestas positivas, seguidos de trastorno afectivo bipolar, déficit de atención y retardo mental, con $83,30 \%$ de respuestas positivas (ver tabla 2 ).

La atención domiciliaria psiquiátrica en el año 2025 incluirá, según los expertos, controles en $100 \%$, atención prehospitalaria en $83,3 \%$, consulta de primera vez en $66,7 \%$ y urgencias en $58,3 \%$; no se anotaron opciones adicionales.

A la afirmación "Se espera que para el futuro los servicios de farmacodependencia deban estar dentro de las instituciones de psiquiatría y hospital psiquiátrico, en su concepto estos": los expertos respondieron:

- "No se integrarán a estas instituciones": sí en $8,33 \%$ y no en $91,67 \%$

- "Se integrarán solo parcialmente: sí en $83,33 \%$ y no en $16,67 \%$

- "Se integrarán a todas las instituciones": sí en $16,67 \%$ y no en $83,33 \%$.

A la afirmación "Algunas personas opinan que los programas específicos de desintoxicación deben estar en instituciones especializadas, algunas por ejemplo en alcohol y otras en otras sustancias psicoactivas o adicciones comportamentales (juego, internet, etc.), en su concepto":

- "Habrá instituciones con múltiples programas" recibió $91,67 \%$ de respuestas positivas y $8,33 \%$ negativa.

TABLA 2. INTERVENCIONES PSIQUiÁtRICAS DOMICILIARIAS, AÑO 2025

\begin{tabular}{|c|c|c|}
\hline Patologías & Sí & No \\
\hline $\begin{array}{l}\text { Adicciones comporta- } \\
\text { mentales }\end{array}$ & $75 \%$ & $25 \%$ \\
\hline $\begin{array}{l}\text { Adicciones farmaco- } \\
\text { lógicas }\end{array}$ & $66,70 \%$ & $33,30 \%$ \\
\hline Ansiedad & $66,70 \%$ & $33,30 \%$ \\
\hline Déficit de atención & $83,30 \%$ & $16,70 \%$ \\
\hline Demencia & $100 \%$ & $0 \%$ \\
\hline Depresión & $91,70 \%$ & $8,30 \%$ \\
\hline Esquizofrenia & $91,70 \%$ & $8,30 \%$ \\
\hline Intentos de suicidio & $66,70 \%$ & $33,30 \%$ \\
\hline $\begin{array}{l}\text { Problemas relacionales } \\
\text { (pareja, familia) }\end{array}$ & $41,70 \%$ & $58,30 \%$ \\
\hline Retardo mental & $83,30 \%$ & $16,70 \%$ \\
\hline Trastornos adaptativos & $50 \%$ & $50 \%$ \\
\hline $\begin{array}{l}\text { Trastorno afectivo } \\
\text { bipolar }\end{array}$ & $83,30 \%$ & $16,70 \%$ \\
\hline $\begin{array}{l}\text { Trastornos de apren- } \\
\text { dizaje }\end{array}$ & $66,70 \%$ & $33,30 \%$ \\
\hline Trastornos de conducta & $58,30 \%$ & $41,70 \%$ \\
\hline $\begin{array}{l}\text { Trastornos de conducta } \\
\text { alimentaria }\end{array}$ & $66,70 \%$ & $33,30 \%$ \\
\hline $\begin{array}{l}\text { Trastornos de la perso- } \\
\text { nalidad }\end{array}$ & $41,70 \%$ & $58,30 \%$ \\
\hline $\begin{array}{l}\text { Trastornos de la perso- } \\
\text { nalidad }\end{array}$ & $58,30 \%$ & $41,70 \%$ \\
\hline Trastornos por estrés & $91,70 \%$ & $8,30 \%$ \\
\hline $\begin{array}{l}\text { Trastornos profundos } \\
\text { del desarrollo }\end{array}$ & $66,70 \%$ & $33,30 \%$ \\
\hline Otros (cuáles) & $0 \%$ & $0 \%$ \\
\hline
\end{tabular}


- “Habrá instituciones especializadas por tipo de adicción" tuvo $66,67 \%$ de respuestas positivas y $33,33 \%$ de respuestas negativas.

- "No habrá instituciones especializadas en adicciones" tuvo $25 \%$ de respuestas positivas y $75 \%$ de respuestas negativas.

La factibilidad y la probabilidad de que la respuesta anotada se diera fueron de 3 en $25 \%$ y 4 en $33,3 \%$.
Los programas de atención comunitaria en psiquiatría en el año 2025 estarán disponibles para esquizofrenia y retardo mental $(91,67 \%)$, seguidos por adicciones farmacológicas, trastorno afectivo bipolar, déficit de atención, depresión, problemas de pareja y problemas de familia con $83,33 \%$; el menor porcentaje de positividad lo tuvo adicciones comportamentales con $50 \%$ (ver tabla 3 ).

Tabla 3. Atención COMUnitaria En PSiQuiatría POR Patologías o PROBLEMAS, AÑo 2025

\begin{tabular}{|c|c|c|c|c|}
\hline Patologías o problemas & Sí & $\%$ & No & $\%$ \\
\hline Esquizofrenia & 11 & $91,67 \%$ & 1 & $8,33 \%$ \\
\hline Retardo mental & 11 & $91,67 \%$ & 1 & $8,33 \%$ \\
\hline Adicciones farmacológicas & 10 & $83,33 \%$ & 2 & $16,67 \%$ \\
\hline Trastorno afectivo bipolar & 10 & $83,33 \%$ & 2 & $16,67 \%$ \\
\hline Déficit de atención & 10 & $83,33 \%$ & 2 & $16,67 \%$ \\
\hline Depresión & 10 & $83,33 \%$ & 2 & $16,67 \%$ \\
\hline Problemas de familia & 10 & $83,33 \%$ & 2 & $16,67 \%$ \\
\hline Problemas de pareja & 10 & $83,33 \%$ & 2 & $16,67 \%$ \\
\hline Demencia & 9 & $75 \%$ & 3 & $25 \%$ \\
\hline Disfunción social & 9 & $75 \%$ & 3 & $25 \%$ \\
\hline Intentos de suicidio & 9 & $75 \%$ & 3 & $25 \%$ \\
\hline Trastornos de aprendizaje & 9 & $75 \%$ & 3 & $25 \%$ \\
\hline Trastornos de conducta & 9 & $75 \%$ & 3 & $25 \%$ \\
\hline Trastornos de conducta alimentaria & 9 & $75 \%$ & 3 & $25 \%$ \\
\hline Trastornos por estrés & 9 & $75 \%$ & 3 & $25 \%$ \\
\hline Trastornos profundos del desarrollo & 9 & $75 \%$ & 3 & $25 \%$ \\
\hline Disfunción laboral & 8 & $66,67 \%$ & 4 & $33,33 \%$ \\
\hline Trastornos adaptativos & 8 & $66,67 \%$ & 4 & $33,33 \%$ \\
\hline Ansiedad & 7 & $58,33 \%$ & 5 & $41,67 \%$ \\
\hline Trastornos de la personalidad & 7 & $58,33 \%$ & 5 & $41,67 \%$ \\
\hline Adicciones comportamentales & 6 & $50 \%$ & 6 & $50 \%$ \\
\hline
\end{tabular}




\section{Componente cualitativo}

Se anotan algunas de las respuestas priorizadas según categorías, siguiendo la matriz de análisis presentada previamente.

Pregunta: ¿Qué estrategias serían útiles para adecuarse en atender el posible incremento de la demanda de la consulta externa de salud mental para el año 2025?

Categorías consideradas: modelo de atención, aspectos económicos, formación de talento humano, especialización de trabajo, consulta privada, leyes, condiciones laborales, demanda de servicios.

\section{Categoría: Modelo de atención}

- Selección de datos contenidos en la categoría

"Estrategias de promoción y prevención en salud mental que incluyan múltiples aspectos".

"Fortalecer la disposición de los profesionales de salud mental en los servicios hospitalarios generales, la consulta ambulatoria y los servicios de salud mental. Fomentar la atención domiciliaria y la modalidad del hospital en casa, fomentar los programas de hospitalización parcial u hospital día y recurrir a la telemedicina para atención por psiquiatría”.

"Mejoría de la atención primaria".

"Hacer más accesible la atención a los pacientes, ubicando centros de atención en muchos puntos de las ciudades. Es decir, evitando la concentración en grandes centros de atención psiquiátrica".

124 "Fortaleciendo la formación de los especialistas en modelos de atención ambulatoria, especialmente en el área de psicoterapias".

"Capacitar al primer nivel en atención básica en salud mental".

"Desarrollo e implementación de la Ley de Salud Mental".

"Desarrollo de programas de psiquiatría social".

"Desarrollo de modelos de atención interdisciplinarios innovadores".

Presencia de psiquiatra en unidades de salud general (centros de salud -unidades de salud Plan Obligatorio).

"Considero que si el actual gobierno y los gobiernos sucesivos logran poner en marcha lo escrito en la Ley 1616 de 2013 o Ley de la Salud Mental en Colombia, aprobada hace un poco más de un año, con toda seguridad se apropiarán los recursos para implementar la infraestructura que permita atender a la población aquejada de trastornos mentales en nuestro país".

"Es necesario cambiar la educación en las universidades, sacar la formación de pregrado centrada en 'enfermedades crónicas' en clínicas psiquiátricas; para pasar a formar a las personas en unidades de salud mental y servicios de urgencias, donde se vean intervenciones en crisis, crisis agudas y poder aprender a ver pacientes por consulta externa. Hay que romper la tendencia actual de hospitalizar todo".

"El trabajo transdisciplinar, las tareas serán segmentadas en cuadrícula. Esto no necesariamente beneficiará al paciente, pero generará mayor eficiencia 
en la prestación de un servicio que se pretenderá aun así integral, aunque segmentado a la manera de línea de ensamblaje. En otras palabras, habrá personal destinado a la evaluación y diagnóstico, otro dedicado a la prescripción de la medicación, otros al seguimiento, otros al enlace con las redes sociales y familiares. La consulta especializada de psiquiatría será reservada al "caso difícil" y el especialista tendrá más un carácter de asesor del equipo, equipo que estará asignado a otras tareas de atención primaria”.

- Descripción: los expertos manifiestan que habrá promoción y prevención en salud mental, hospital en casa, telemedicina, consulta ambulatoria y domiciliaria, accesibilidad, red de servicios en comunidad, psiquiatría social, nuevos modelos interdisciplinarios y transdisciplinarios, mayor presencia del psiquiatra en servicios de salud, intervención en crisis, especialización de la atención en sistema de línea de ensamblaje, especialización de la psiquiatría en "casos difíciles".

- Relación entre variables: hay aspectos de la educación profesional, trabajo en equipo y dispositivos con énfasis en Atención Primaria en Salud (APS), modelo comunitario y atención prehospitalaria y posthospitalaria.

- Revisión de los datos: existen elementos de promoción y prevención (PYP), modelo comunitario y dispositivos ambulatorios, domiciliarios, trabajo en equipo, el papel del psiquiatra en comunidad, y el rol del hospital general y los servicios psiquiátricos.

- Posibles explicaciones: existe un direccionamiento de la psiquiatría hacia la atención en comunidad, lo cual, si bien no se ha realizado, se erige como un ideal de atención.

- Conceptualización más general (patrón cultural): hay barreras de la atención y modelo de APS, comunidad y transdisciplinariedad, dado el sistema de salud de Colombia.

\section{Categoría: aspectos económicos}

- Selección de datos contenidos en la categoría

"Ahora bien, la demanda de consulta externa de salud mental hace inmediata alusión a una forma de operar dentro del sistema de prestación de servicios, en este caso la regulación de la oferta irá obviamente respondiendo a los intereses del prestador. Asumo que esto va a generar una práctica alternativa en salud mental que se reserva a quienes tienen poder adquisitivo suficiente para pagarlo, y en este caso estamos hablando de un incremento de la consulta privada".

"[...] con toda seguridad se apropiarán los recursos para implementar la infraestructura que permita atender a la población aquejada de trastornos mentales en nuestro país".

"En cuanto a la atención de las aseguradoras que se sensibilicen, disminuya el estigma, vean los beneficios (integrales, económicos etc.) de la atención en salud mental".

- Descripción: los expertos hacen evidente el modelo de prestación de servicio actual, del aseguramiento en salud, la necesidad de recursos y ampliación de beneficios.

- Relación entre variables: hay opiniones encontradas en cuanto al aseguramiento 
de los recursos para la atención, ligados a cambios en comprensión de los beneficios de tratar adecuadamente la enfermedad mental.

- Revisión de los datos: se establece que el libre mercado y el modelo actual de prestación van ligados a un beneficio de los aseguradores, haciendo necesario el cambio de comprensión de estos sobre los beneficios de tratar la enfermedad mental, incluyendo los beneficios económicos.

- Posibles explicaciones: hay un debate actual sobre el papel de los aseguradores en la atención en salud en general y los alcances y limitaciones de la prestación de servicios de salud; uno de los aspectos más resaltados es el uso de recursos específicamente para salud y no desviaciones de estos por intereses particulares.

\section{Categoría: formación de talento humano}

\section{- Selección de datos contenidos en la categoría}

"Fortaleciendo la formación de los especialistas en modelos de atención ambulatoria, especialmente en el área de psicoterapias".

"Estimular la especialización en psiquiatría para médicos generales, capacitar al primer nivel en atención básica en salud mental".

"Desarrollo e implementación de la ley de salud mental, capacitación del personal médico y paramédico al respecto [...] divulgación, psicoeducación a la población".

"Es necesario cambiar la educación en las universidades, sacar la formación de pregrado centrada en 'enfermedades crónicas' en clínicas psiquiátricas, para pasar a formar a las personas en unidades de salud mental y servicios de urgencias".

- Descripción: los expertos anotan la necesidad de formación de especialistas en aspectos de atención ambulatoria, psicoterapias, formación de médicos generales en salud mental, en pregrado en el campo amplio de la psiquiatría, a profesionales del primer nivel en salud mental y la divulgación y psicoeducación del público en general.

- Relación entre variables: se aprecia necesidad de realizar acciones de cambio en la formación de personal profesional y no profesional a diferentes niveles, desde el público en general a los especialistas en psiquiatría.

- Revisión de los datos: es interesante que se prevén cambios no solo en la psiquiatría como especialidad, sino también en la formación de diferentes disciplinas en pregrado y capacitación a los usuarios.

- Posibles explicaciones: actualmente hay fallas en la formación de especialistas en aspectos de psiquiatría comunitaria, en la integración de la especialidad al trabajo con otras disciplinas y que la salud mental no es énfasis en programas de pregrado.

- Conceptualización más general (patrón cultural): al momento hay incluso Instituciones de Educación Superior que en medicina no tienen rotación de psiquiatría, aduciendo la autonomía universitaria; la psiquiatría no es requisito de formación de talento humano en pregrado. 
- Formulación tentativa de nuevas hipótesis: la respuesta a los problemas de salud mental no solo tiene que ver con el incremento de infraestructura física, también se debe formar adecuadamente al talento humano en salud mental.

Pregunta: ¿Qué estrategias considera usted que deben ser implementadas para atender el incremento previsto en la demanda de atención primaria en salud mental?

Categorías consideradas: infraestructura y logística, capacidad instalada, consulta ambulatoria.

\section{Categoría: infraestructura y logística}

\section{- Selección de datos contenidos en la categoría}

"Mejoría de los centros".

"Crear centros comunitarios de salud mental”.

"Sitios".

"Desarrollo de más centros de atención en todo el país".

"Consultorio de psiquiatría en los centros, unidades de salud de primer nivel...".

"Es necesario la creación de infraestructura física anexa a los sitios de atención primaria, en los que pueda brindarse apoyo especializado a los médicos generales en la atención de los pacientes que tienen patología psiquiátrica".

"No habrá un incremento de las camas de psiquiatría, pero se conservarán las actuales para el manejo de crisis.
En cambio, sí se requiere adecuar los espacios para la atención en consulta externa, espacios que aún son insuficientes, con poca privacidad o pensados para un proceso eminentemente médico, no para el acto comunicacional que implica una consulta”.

- Descripción: la apuesta de los expertos se centra en más servicios, más cerca de la comunidad y unidades de primer nivel.

- Relación entre variables: se establece la necesidad de más centros, con mayor cobertura y personal de APS.

- Revisión de los datos: no hay una adecuada descripción de cómo deberían ser los centros, aunque sí se anota la necesidad de ellos.

- Posibles explicaciones: el modelo actual enfatiza en la hospitalización, hay falta de personal e instituciones para la atención en comunidad.

Pregunta: servicios especiales en hospitalización.

Categorías consideradas: cambio de imagen y enfoque, creación de servicios, neurociencias, sostenibilidad económica, terapias grupales, incertidumbre, redes de servicios, adecuación "en la marcha”.

\section{Categoría: cambio de imagen y enfoque}

- Selección de datos contenidos en la categoría

"Hacer desaparecer su carácter manicomial o represivo. Cambiar su carácter declarado de tipo confesional y caritativo al que está históricamente vinculado. Incorporar decididamente 
el concurso de las neurociencias y de las terapias grupales, tanto para diagnóstico y evaluación, como para intervención temprana que incorpore aspectos relacionales; son estos últimos los que van a ser útiles para evitar la rehospitalización".

"Que los crónicos y discapacitados no estén en psiquiatría”.

"La Atención primaria en Salud es necesaria, pero su existencia no implica la desaparición o reducción de los servicios de hospitalización, que siempre serán requeridos con enfoques de suficiencia para la atención adecuada y oportuna de los trastornos agudizados. Es necesario el enfoque de hospitalización con metas de estancias cortas. El enfoque administrativo debe basarse en la sostenibilidad financiera, la eficiencia de las instituciones y la efectividad de los tratamientos, reduciendo la mayor relevancia del valor social, en el cual se ha pretendido escudar la falta de sostenibilidad o productividad de los servicios".

"Acercarse más a la comunidad o diseñar acciones para acercar a la comunidad al hospital".

"Mejorar ampliamente la atención ambulatoria, generar prevención primaria en psiquiatría".

- Descripción: la referencia al manicomio sigue presente en las descripciones de la psiquiatría, al igual que los aspectos de beneficencia, caridad y confesionalidad de las instituciones. La cronificación de pacientes, por un lado, y los abordajes de atención primaria en salud mental (APS) son los dos polos del espectro de la acción de la psiquiatría en la segunda década del siglo XxI.
- Relación entre variables: hay una necesidad expresada de cambio de campo de acción y modelo que se enfrenta a la existencia de personas a las cuales no se les da respuesta en otras instancias. Los aspectos de sostenibilidad financiera, adopción de modelo APs, tratamiento de agudos en forma eficaz son discursos de la psiquiatría actual.

- Revisión de los datos: hay una apuesta por la intervención temprana y el abordaje sistémico relacional para evitar el deterioro y la cronificación, no tanto basado en aspectos del tratamiento en sí (farmacológico y no farmacológico), además de una lucha de la psiquiatría como campo legítimo de ciencia médica.

- Posibles explicaciones: se debe aplicar un modelo curativo, aunque haya necesidad de un modelo preventivo y rehabilitador, dada la limitación de las instituciones y el sector salud.

- Conceptualización más general (patrón cultural): se aprecia que la psiquiatría hospitalaria sigue siendo dominada por la institución tipo manicomial, con una búsqueda de mayor aceptación.

- Formulación tentativa de nuevas hipótesis: los expertos consultados siguen evaluando la institución psiquiátrica con el referente de la institución asilar, lo cual puede limitar las actuaciones en psiquiatría, particularmente para las patologías crónicas.

\section{Categoría: creación de servicios}

- Selección de datos contenidos en la categoría 
"Especializarse en programas de atención específicos".

"Considero que son más útiles las unidades de salud mental anexas a los hospitales generales".

"Crear, mantener, financiar servicios ambulatorios de consulta externa, unidades de salud mental, centros comunitarios para enfermedades crónicas y servicios de tratamientos ambulatorios domiciliarios específicos en el área de salud mental y psiquiatría. Para realizar manejos más integrales, biopsicosociales en la patología psiquiátrica desde una perspectiva más construccionista".

- Descripción: los expertos consultados están de acuerdo con la creación de programas específicos de atención para patologías o condiciones particulares, unidades de salud mental en hospital general, servicios de consulta externa, unidades de salud mental, centros comunitarios para enfermedades crónicas y servicios de atención domiciliaria.

- Relación entre variables: se manifiesta la necesidad de aumentar el portafolio de servicios de atención psiquiátrica, incluyendo servicios especializados, unidades de salud mental, consulta externa y atención domiciliaria.

- Revisión de los datos: se aprecia la necesidad de incrementar el campo de acción a servicios que no son claramente prestados en la actualidad desde la psiquiatría, como los servicios especializados por patología, limitados a adicciones, trastornos de la alimentación, gerontopsiquiatría, paidopsiquiatría y atención por patologías psiquiátricas, los cuales se tienen solo en algunas clínicas psiquiátricas; los centros comuni- tarios tienen dificultad en ser asumidos por el sector salud, así como los costos y limitaciones de montar un servicio de atención domiciliaria en psiquiatría, limitado actualmente a atención de urgencia para traslado a los centros.

- Posibles explicaciones: el modelo actual en teoría incluye la rehabilitación y reinserción social del enfermo, pero estas acciones son insuficientes desde las limitaciones del sector salud, específicamente para problemas tales como el desarrollo de servicios comunitarios, apartamentos protegidos, centros día, etc. La atención domiciliaria se limita actualmente por reglamentación y exigencias de seguridad, dispensación de medicación, etc.

- Conceptualización más general (patrón cultural): los expertos desean una atención en psiquiatría con desarrollo de servicios, modernización de atención y ampliación de cobertura, aunque en la actualidad hay múltiples limitaciones.

- Formulación tentativa de nuevas hipótesis: el modelo de atención es el de psiquiatría general, con una necesidad de desarrollo de otros modelos y servicios que no siempre pueden ser viables económicamente, como la atención domiciliaria y la atención de pacientes crónicos

\section{Implementación de atención domiciliaria}

Pregunta: ¿Cómo deberían los psiquiatras y las instituciones implementar ese servicio?

Categorías consideradas: redes y servicios, territorio, guías, formación, plan de beneficios, medición, referenciación. 
Categoría: redes y servicios.

\section{- Selección de datos contenidos en la categoría}

"Se debería tener una formación específica y certificación en el modelo de medicina domiciliaria, y contar con la red de apoyo institucional, un equipo de salud mental para la visita, y con los diferentes niveles de atención: consulta externa, hospital de día, urgencias y unidad de salud mental".

"Brindando las condiciones logísticas y de equipos para la prestación de este servicio".

"Es importante definir extensión hospitalaria y atención domiciliaria. El primero, como su nombre lo indica, es llevar los cuidados hospitalarios al domicilio, y lo segundo es brindar los cuidados médicos ambulatorios en el domicilio para disminuir la demanda de atención en los lugares de mayor afluencia como las urgencias generales. La atención ambulatoria permite un contacto más cercano y en colaboración con el grupo familiar e incluso con la comunidad".

"Ambulancias, visitas periódicas de equipo interdisciplinario, evaluación de resultados riesgo-beneficio, etc.".

"Aumentar contratación y proyectos sobre esta modalidad".

"Constituyendo equipos interdisciplinarios extramurales que realicen su desplazamiento para la atención fuera del consultorio y en forma simultánea o secuencial".

"Diseño juicioso de los programas que es preciso desarrollar, con personal suficiente, bien capacitado y bien remunerado".

- Descripción: hay referencia a redes de profesionales y atención en consulta externa, hospital de día, urgencias y unidades de salud mental, así como modelos de extensión hospitalaria y atención domiciliaria. Se establece que hay necesidad de ambulancias y equipo profesional capacitado, disponible para desplazamientos y con condiciones salariales adecuadas.

- Relación entre variables: la implementación de este tipo de atención parece considerarse principalmente una extensión y complemento de los servicios existentes en urgencias, hospitalización y servicios ambulatorios. Hay necesidad de recurso humano, transporte, ambulancias y capacitación específica.

- Revisión de los datos: los expertos manifiestan la necesidad de desarrollar este tipo de atención, aunque señalan aspectos de seguridad del personal y accesibilidad.

- Posibles explicaciones: no hay adecuada georreferenciación, el modelo actual es por aseguradoras, cuya población está dispersa; no hay equipos que desarrollen esta actividad en forma exclusiva, siendo parte de las actividades desarrolladas en urgencias, hospitalización o consulta externa. No hay a la fecha personal suficiente entrenado en este tipo de modelo y atención.

- Conceptualización más general (patrón cultural): la psiquiatría está ligada a la institución psiquiátrica, el desarrollo de estos servicios se ve limitado por la cobertura, los costos y los riesgos. 
- Formulación tentativa de nuevas hipótesis: hay una necesidad de desarrollar esta actividad, pero al momento no hay alternativas adecuadas.

- Comentarios: en referenciación los expertos consideraron experiencias nacionales e internacionales de aplicación de modelo.

\section{Categoría: territorio}

- Selección de datos contenidos en la categoría

"(Modelo por territorios): georreferenciación de los casos, pero también de los servicios, para que los operadores conozcan el territorio, las redes existentes y recursos de la zona. Toma de decisiones colegiadas y comité de seguimiento de los casos".

"Por agenda programada y bajo la premisa de referenciación geográfica”.

- Descripción: la aplicación del modelo de atención domiciliaria se ve ligada a un sistema de georreferenciación de usuarios.

- Relación entre variables: en otros puntos, los expertos anotan la necesidad de equipos, sistemas de transporte y seguimiento.

- Revisión de los datos: los expertos anotan la necesidad de territorio asociada al desarrollo de modelo, no solo de atender en casa, también el poder realizar seguimiento al caso y el manejo de este por equipo estable.

- Posibles explicaciones: hay limitaciones en la aplicación del modelo, el cual puede no ser costo-efectivo, ni tampoco eficaz en términos de intervención dentro de un modelo que asegure la continuidad de atención.

- Conceptualización más general (patrón cultural): el referente de atención en salud es la institución. Partiendo de ella, es necesario optimizar el servicio en un radio de acción para asegurar la accesibilidad, los costos y la continuidad de cuidados. La atención domiciliaria es a la fecha una actividad, no un modelo.

- Formulación tentativa de nuevas hipótesis: el énfasis parece haberse puesto en equipos que aseguren el desplazamiento domiciliario y un abanico de posibilidades de atención posteriores.

Categoría: guías

- Selección de datos contenidos en la categoría

"Deben implementarse guías de gestión de caso psicosocial (entendiendo por caso no el individuo, sino los problemas que han sido definidos como tales y que rara vez se limitan a la salud mental)".

"Con protocolos para definir que paciente se beneficia y cuál es el objetivo de la visita domiciliaria".

"Con protocolos de atención, seguimiento, ...”

- Descripción: se menciona la necesidad de guías y protocolos, específicamente guías de caso psicosocial que incluyan seguimiento de caso.

- Relación entre variables: se aprecia la necesidad de definir claramente el tipo 
de población que se va a atender, la responsabilidad con respecto al caso y el seguimiento.

- Revisión de los datos: por un lado, hay necesidad de protocolos para atención claros; de otra parte, las guías de gestión de caso.

- Posibles explicaciones: en la actualidad la atención se hace sobre caso "difícil” y por necesidades de tipo social, más que por indicación clínica.

- Conceptualización más general (patrón cultural): el modelo de gestión en salud y los procesos de habilitación y acreditación llevan a los psiquiatras a pensar en la necesidad de definiciones y estandarización de procesos.

- Formulación tentativa de nuevas hipótesis: se piensa en modelos de tipo administrativo y de MBE para asegurar el manejo adecuado de casos.

\section{- Búsqueda de nuevos hallazgos en los} datos: al momento, no hay buen nivel de evidencia de manejo de casos en atención domiciliaria, pero hay necesidad de guías de actuación para evitar la dispersión de acciones, mantener el foco terapéutico y estandarizar las actuaciones.

\section{Discusión}

La metodología Delphi es dispendiosa, los autores encontraron que a pesar de que la tecnología se puede utilizar en su favor, la participación no se logró en la medida solicitada; es posible que esto fuera por lo extenso del cuestionario, la falta de familiaridad con las encuestas y la pobre motivación de participar; encontramos que algunos expertos abrieron el aplicativo, pero no respondieron.
Se requirió motivar la participación en forma telefónica y por correos electrónicos, por lo cual el cegamiento se debió romper. Pese a lo anterior, los autores intentaron mantener el anonimato y cegamiento, realizando una asignación de código en las respuestas para el componente cualitativo y dando igual peso a todas las respuestas. El sistema de encuesta por internet es de fácil uso, poco costo y permite el seguimiento permanente de las encuestas.

Existe gran incertidumbre de adecuación de la institución psiquiátrica en el futuro; al momento de la investigación se hundió el proyecto de reforma a la salud y la ley de salud mental no estaba implementada. Existe gran expectativa por la implementación y los efectos de la Ley 1616 de 2013 (12), reflejada en una mayor implementación de atención en salud mental a la comunidad, con incremento en promoción y prevención y el desarrollo de equipos de profesionales con competencias específicas en salud mental. La incertidumbre del futuro de la institución psiquiátrica se asocia a que en Colombia, las sucesivas reformas de salud no han logrado corregir las inequidades, incluyendo un deterioro de la salud mental en la política pública (5), y a leyes específicas, pero ineficaces en la práctica. Aspectos como la transición epidemiológica y los cambios en la composición de la población acentuarán las inequidades, a menos que existan políticas de salud mental efectivas que incluyan una mayor cobertura de programas y servicios (26).

Si bien se reconoce la necesidad de cambio y adecuación de la institución psiquiátrica en la próxima década, no es clara la forma de realizarla. Se enfatiza en la educación de equipo, incluyendo profesionales no psiquiatras, mejoría en infraestructura e incremento de servicios, incluyendo consulta, hospitalización total y parcial, clínicas específicas y atención comunitaria. 
Los expertos consultados mostraron un consenso acerca del incremento al año 2025 de programas como consulta externa, atención comunitaria y atención domiciliaria, en mayor proporción que la atención hospitalaria y la hospitalización parcial.

La consulta externa por psiquiatría se prevé que aumentará en forma importante (más del $50 \%$ ), principalmente en patologías tales como adicciones, depresión, ansiedad, trastornos de conducta alimentaria, trastornos por estrés y trastornos bipolares. Esta tendencia es apoyada por la literatura y proyecciones de la ops (17). Se considera, adicionalmente, un incremento en la hospitalización en psiquiatría a expensas de los citados diagnósticos. Un $40 \%$ de los encuestados prevén que la consulta de esquizofrenia por psiquiatra disminuirá y que profesionales no psiquiatras asumirán gran parte del cuidado en atención domiciliaria.

APS en salud mental: se debe explorar el campo de APS en salud mental, dependiente de mejoras en modelos, infraestructura y formación de recurso humano. A la fecha de la encuesta, los planes de pyp en salud mental no están desarrollados, no hay claridad de quién deba asumir su implementación. Existe pobre definición y acuerdo sobre la atención comunitaria, la cual es considerada importante, pero más allá del campo actual de la psiquiatría, como parte del sector social pobremente integrado al de salud en la actualidad.

Unidades de salud mental en hospital general: se prevé un incremento de estos servicios para el año 2025, con atención a cualquier tipo de patología o condición, con perfil similar al del hospital psiquiátrico.

Clínica psiquiátrica: la evolución de la hospitalización psiquiátrica mostró que hay incertidumbre sobre su evolución al año
2025; no se logró consenso, las respuestas incluyeron una posible disminución moderada o leve, permanecer igual o un incremento leve, moderado o mayor al $100 \%$.

En cuanto a la opinión sobre la atención hospitalaria en salud mental en clínica psiquiátrica tampoco se logró consenso, los expertos incluyeron todos los problemas listados en un rango amplio de problemas psiquiátricos y relacionales, tanto agudos como crónicos.

Servicios especializados: los expertos consideran que los programas especializados para farmacodependencia, larga estancia, trastornos de la conducta alimentaria, inimputabilidad y retardo mental se deberían considerar. Esto parece estar ligado a la epidemiología de los trastornos y a la demanda actualmente no satisfecha de atención, en particular de población con problemas crónicos, repercusiones legales y familiares.

Crónicos: hay escepticismo de que el Estado se haga cargo de las condiciones sociales, serán previsiblemente asumidas por instituciones públicas, privadas y mixtas; esto cobra mayor importancia, teniendo en cuenta el proceso de paz con la guerrilla de las FARC y las necesidades del posconflicto que se están discutiendo.

Hospitalización parcial: los expertos no concuerdan en la importancia de la hospitalización parcial en el modelo de atención en el futuro, ligado a las expectativas de integración social del enfermo mental, lo cual refleja que la hospitalización psiquiátrica en Colombia no es un servicio intermedio entre la hospitalización total y la comunidad (27), dada la falta de integración del sector salud y la comunidad.

Institución ideal: la institución ideal para los psiquiatras incluye los servicios que actualmente se están ofertando en agudos, 
poblaciones de inimputables, rehabilitación en farmacodependencia, así como clínicas específicas por patologías, redes de servicios, telepsiquiatría, centro de empleo, etc. Parecería que, basados en la institución actual, se pretende crear un agregado de servicios que den respuesta a condiciones que actualmente se atienden y otras que se asumen. Llama la atención que se nombra, por un lado, la necesidad de modernizar la institución y, por otro lado, de mantener la atención a pacientes crónicos, institucionalizados por condiciones psicosociales.

La atención a pacientes crónicos, inimputables, con retardo mental y demencia debería ser responsabilidad estatal, pero también se ubica en la institución ideal, lo cual evidencia las dificultades de intervención en los problemas sociales y familiares asumidos por el sector salud. Esta situación no parece que vaya a mejorar en la siguiente década, aunque el rol de la familia es fundamental en la reforma de la atención psiquiátrica, de cara a la desinstitucionalización progresiva, como fue resaltado por los expertos (28).

Atención domiciliaria: la opinión de los expertos con respecto al desarrollo de la atención domiciliaria en salud mental prevé que aumentará, aunque su desarrollo no es claro. Las intervenciones más susceptibles de ser desarrolladas en esta modalidad de atención se centrarán en depresión, trastorno por estrés, esquizofrenia, bipolaridad, déficit de atención y retardo mental. La atención domiciliaria se centrará en los controles y la atención prehospitalaria.

Si bien la investigación se centró en la institución psiquiátrica, las opiniones de los expertos son acordes con recomendaciones de la OMs para la salud mental, las cuales incluyen el fortalecimiento de la atención en el nivel 134 primario, la atención en la comunidad y la educación al público, involucrando a las co- munidades, las familias y los consumidores, así como políticas, programas y legislación nacionales efectivas, el desarrollo del recurso humano y la vinculación otros sectores (17).

La opinión de los expertos consultados concuerda con los principios de atención en salud mental referidos en reformas de atención en países desarrollados, como son integración, centralización de cuidados específicos, cuidados compartidos con personal no psiquiátrico, intervención temprana, continuidad de cuidados, unidades especializadas, manejo del uso de servicios y aseguramiento de la calidad (29).

\section{Agradecimientos}

A los expertos consultados Alfonso Rodríguez (Universidad El Bosque), Álvaro Osorio (Clínica Nuestra Señora de La Paz), Carlos Filizzola (Universidad Javeriana), Gabriel Oviedo (Hospital de San Ignacio), Henry García (Clínica Montserrat), Jimena Mayorga (Clínica Nuestra Señora de La Paz), Luis Eduardo Jaramillo (Universidad Nacional), Miguel Cote (Universidad Nacional), Ricardo Yamín (Universidad del Rosario), Ronald Prado (Hospital Simón Bolívar) y Victoria Carrascal (Clínica Montserrat).

\section{Referencias bibliográficas}

1. Oficina del Alto Comisionado para los Derechos Humanos (ACNUDH) -Organización Mundial de la Salud (oms). Derechos humanos, salud y estrategias de reducción de la pobreza. Serie de Publicaciones sobre Salud y Derechos Humanos, Número 5; 2008.

2. Organización Mundial de la Salud (oms). Plan de Acción Sobre Salud Mental 2013-2020. Ginebra: oMs; 2013.

3. Organización de las Naciones Unidas (ONU). Objetivos de Desarrollo del Milenio. Informe 2014. Nueva York: onU; 2014.

4. Organización Mundial de la Salud (oms). 65 Asamblea Mundial de la Salud. Punto 132. Carga mundial de trastorno mental y necesidad de que el 
sector de la salud y el sector social respondan de modo integral y coordinado a escala de país; 2012.

5. Lozano J, Ardila S. Marco legal y psiquiatría: el ejemplo colombiano. En: Gómez C, Hernández G, Rojas A, Santacruz H y Uribe M, editores. Psiquiatría clínica. Diagnóstico y tratamiento en niños, adolescentes y adultos, tercera edición. Bogotá: Editorial Médica Panamericana; 2008.

6. Comité de Políticas Públicas de la Asociación Colombiana de Psiquiatría. Política Nacional del Campo de la Salud mental: entre el escepticismo y la esperanza (editorial). Revista Colombiana de Psiquiatría. 2008; 37 (3).

7. Maya E. El derecho a la salud en la perspectiva de los derechos humanos y del sistema de inspección, vigilancia y control de quejas en materia de salud (editorial). Revista Colombiana de Psiquiatría. 2008; 37 (4).

8. United Nations Educational, Scientific and Cultural Organization (Unesco). Declaración Universal sobre Bioética y Derechos Humanos; 2005.

9. Posada J. La salud mental en Colombia y la Ley 100; oportunidades y amenazas. Revista Colombiana de psiquiatría. 2003; 32 (Supl 1): 6-12.

10. Organización Panamericana de la Salud (OPS). Declaración de Caracas [internet], 14 de noviembre de 1990 [acceso: 30 de mayo del 2014]. Disponible en: http://www.psi.uba.ar/academica/carrerasdegrado/ psicologia/sitios_catedras/electivas/067_psico_preventiva/cursada/dossier/declaracion_caracas.pdf.

11. Ministerio de la Protección Social, Fundación FEsSocial. Lineamientos de Política de Salud Mental para Colombia. Cali: Gráficas Ltda.; 2005.

12. República de Colombia. Ley 1616 de 21 de enero de 2013, "por medio de la cual se expide la ley de Salud mental y se dictan otras disposiciones" [internet] [acceso: 12 de agosto del 2015]. Disponible en: http://wsp.presidencia.gov.co/Normativa/Leyes.

13. Documento Propuesta de ajuste de la Política Nacional de Salud Mental para Colombia 2014 [internet]. Disponible en: http://puj-portal.javeriana.edu.co/portal/pls/portal/docs/1/5304073.PDF.

14. Posada J. La salud mental en Colombia. Biomédica Revista del Instituto Nacional de Salud. 2007; 33 (4).

15. ops/OMs. 35a Sesión del Subcomité del Comité Ejecutivo de Planificación y Programación. La salud mental en las Américas, nuevos retos al comienzo del nuevo milenio 2001. Washington: ops; 2000.
16. Posada JA, Aguilar SA, Magaña CG, et al. Prevalencia de trastornos mentales y uso de servicios: resultados preliminares del Estudio Nacional de Salud Mental. Colombia 2003. Rev Colomb Psiquiatr. 2004; (33): 241-62.

17. Rodríguez JJ, editor. La reforma de los servicios de salud mental: 15 años después de la Declaración de Caracas. Washington, D.C: ops; 2007.

18. Rosselli H. Historia de la psiquiatría en Colombia. Bogotá: Editorial Horizontes; 1968.

19. Basaglia F. La institución negada. Informe de un hospital psiquiátrico. Buenos Aires: Barral Editores; 1972.

20. González L. Administración en salud mental. En: Gómez C, Hernández G, Rojas A, Santacruz H, Uribe M, editores. Psiquiatría clínica. Diagnóstico y tratamiento en niños, adolescentes y adultos, tercera edición. Bogotá: Editorial Médica Panamericana; 2008.

21. Arias J. ¿Hacia dónde van los modelos de atención médica? Revista Conexxión, Acemi. 2014: 3 (7)

22. República de Colombia. Constitución Política de 1991 [internet]. Disponible en: http://www.unesco. org/culture/natlaws/media/pdf/colombia/colombia_constitucion politica_1991_spa_orof.pdf, 1991.

23. República de Colombia. Ley 100 de 21 de enero de 2013 [internet] [acceso: 10 de noviembre del 2013]. Disponible en: www.alcaldiabogota.gov.co/norma/.

24. Astigarraga E. El método Delphi. San Sebastián, España: Universidad de Deusto, Facultad de Ciencias Económicas y Empresariales; 2000.

25. Carreño M. El método Delphi: cuando dos cabezas piensan más que una en el desarrollo de guías de práctica clínica. Rev Colomb Psiquiat. 2009; 38 (1).

26. Kohn R, Levav I, Caldas de Almeida JM, Vicente B, Andrade L, Caraveo-Anduaga JJ, Saxena S, Saraceno B. Los trastornos mentales en América Latina y el Caribe: asunto prioritario para la salud pública. Rev Panam Salud Pública. 2005; 18 (4/5): 229-40.

27. Ministerio de Sanidad y Política Social de España. Hospital día. Estándares y recomendaciones. Madrid; 2009.

28. Ardila S. El apoyo familiar como uno de los pilares de la reforma de la atención psiquiátrica. Consideraciones desde una perspectiva psicosocial. Revista Colombiana de Psiquiatría. 2009; 38 (1).

29. Ministry of Health and Ministry Responsible for Seniors. British Columbia. B.C.'s Mental Health Reform. Best Practices for Inpatient/outpatient services. 